\title{
Coexisting Tetrahedrite-(Zn) and Sphalerite at the Teremki Gold-Ore Deposit (East Transbaikalia): Chemical Composition and Formation Conditions
}

\author{
N. G. Lyubimtseva ${ }^{a, *}$, V. Yu. Prokof'ev ${ }^{a}$, and N. S. Bortnikov ${ }^{a}$ \\ ${ }^{a}$ Institute of Geology of Ore Deposits, Petrography, Mineralogy, and Geochemistry, \\ Russian Academy of Sciences, Staromonetnyi per., 35, Moscow, 119017 Russia \\ *e-mail: luy-natalia@yandex.ru \\ Received March 5, 2021; revised May 30, 2021; accepted June 7, 2021
}

\begin{abstract}
Paragenetic associations of tetrahedrite-( $\mathrm{Zn})$ and sphalerite are distinguished in the Teremki goldore deposit. The chemical composition of coexisting minerals of this association is determined. The $\mathrm{Sb} /(\mathrm{Sb}+$ $\mathrm{As})$ and $\mathrm{Fe} /(\mathrm{Fe}+\mathrm{Zn})$ ratios in tetrahedrite $-(\mathrm{Zn})$ vary from 0.66 to 0.97 and from 0.28 to 0.40 , respectively. A negative correlation was established between $\mathrm{Sb} /(\mathrm{Sb}+\mathrm{As})$ and $\mathrm{Fe} /(\mathrm{Fe}+\mathrm{Zn})$ ratios. Contents of Fe in sphalerite change from 0.88 to $1.43 \mathrm{wt} \%(1.5-2.5 \mathrm{~mol} \% \mathrm{FeS})$. Temperature and sulfur fugacity when precipitation of tetrahedrite-( $\mathrm{Zn})$-paragenesis were estimated: they range from 130 to $280^{\circ} \mathrm{C}$ and from $10^{-13.2}$ to $10^{-8.1}$ bars, respectively.
\end{abstract}

Keywords: Teremki deposit, tetrahedrite-(Zn), sphalerite, coexisting minerals, Fe and Zn distribution, geothermometer, formation condition

DOI: $10.1134 / \mathrm{S} 1075701521050032$

\section{INTRODUCTION}

One of the key issues in present-day mineralogy, geochemistry, and studies of ore deposits is to reconstruct the mineral-formation conditions and reveal the regularities of the element distribution between coexisting minerals. When solving the ore genesis problems, mineral solid solutions, where the ratios between elements are determined by the physicochemical parameters of the mineral-forming fluid and the behaviour of their distribution between the minerals, are of considerable interest.

The objects of the present study are coexisting tetrahedrite and sphalerite from the Teremki gold-ore deposit (East Transbaikalia). These two sulfides demonstrate broad variations in their compositions, are often associated with each other, and their chemical compositions (together with its variations) are often used as an indicator of the physicochemical conditions of ore formation. The applicability range of research on the mineral association of tetrahedrite and sphalerite for estimating temperatures has been broadened owing to studies on thermodynamic properties of these minerals (Raabe and Sack, 1984; Sack and Loucks, 1985; Sack, 2017; etc.).

The Teremki deposit is located $5 \mathrm{~km}$ from the large Darasun gold deposit. Mineral assemblages at these deposits are similar, although assemblages at the Teremki deposit have not been studied in the same detail as those of the Darasun deposit (Timofeevskii, 1972; Prokof'ev et al., 2004; Lyubimtseva et al., 2018a, $2018 \mathrm{~b}$; etc.). The formation conditions of the productive stage at the Teremki deposit have been determined from the study of fluid inclusions in quartz (Prokof'ev et al., 2004; Prokofiev et al., 2010), whereas ore minerals of variable composition (e.g., tetrahedrite and sphalerite, which may be associated with native gold) have not been studied in this regard.

\section{BRIEF DESCRIPTION OF THE DEPOSIT}

The Teremki deposit is located in Chita region, near the settlement of Vershino-Darasunskii (Figs. 1a, 1b). The deposit consists of a series of gently sloping auriferous tourmaline-quartz sulfide ores and disseminated ore zones. It is related to a fault system, on the one hand, and to granodiorite-porphyry of the Amudzhikan Complex $\left(\mathrm{J}_{2}-\mathrm{K}_{1}\right)$, on the other; the host rocks are Middle Paleozoic-Early Mesozoic gabbroids $\left(\mathrm{Pz}_{1}\right)$ and granitoids (Timofeevskii, 1972; Prokof'ev et al., 2004; Prokofiev et al., 2010; etc.) (Fig. 1c). Mineralization of gold-ore veins formed in three stages: (1) early one (quartz, tourmaline, sericite, pyrite, arsenopyrite, and chalcopyrite), (2) productive (quartz, pyrite, chalcopyrite, sphalerite, fahlore, galena, marcasite, native gold, native silver, pyrrhotite, bournonite, native bismuth, bismuthite, matildite, aikinite, cosalite, tetradymite, and others), and 
(3) post-mineral (quartz and carbonate). Native gold grains of are rounded, with smooth boundaries, up to $50 \mu \mathrm{m}$ in size, intergrown with quartz, chalcopyrite, sphalerite, galena, and bismuth minerals. In some veins, a change of quartz-tourmaline and quartzpyrite assemblages at selvages to quartz-sulfide paragenesis with native gold in intermediate zone and to quartz-carbonate aggregates in the central parts of the veins was observed. Brecciated quartz-tourmaline and pyrite fragments are often cemented with sulfides.

\section{MINERAL RELATIONSHIPS}

Ore minerals at the Teremki deposit are represented by fragments of monomineralic grains and sulfide intergrowths $1-2 \mathrm{~mm}$ or less in size, cemented with carbonate and disseminated in the host rock (Figs. 2a, 2b).

Microscopic investigation of the mineral relationships has shown that tetrahedrite and sphalerite, together with galena, chalcopyrite, and carbonate, fill interstices between quartz grains. Galena-chalcopyrite-tetrahedrite aggregates cement broken grains of early pyrite (Fig. 2c) and form coarse-grained carbonate-cemented aggregates (Figs. 2e, 2f). Relationships between tetrahedrite and chalcopyrite in these aggregates are highly variable. There are not only intergrowths with mutually smooth boundaries between grains of these minerals (Figs. 2e, 2f, 2h, 3b, 3d), but also tetrahedrite veinlets cross-cutting chalcopyrite (Figs. 2h, 3c) can be found. Sphalerite-galena-chalcopyrite-tetrahedrite aggregates form coarse-grained aggregates in carbonate (Figs. 2g, 3c), and, together with carbonate, fill interstices between quartz grains (Fig. 3d), while fine grains of these aggregates are cemented with carbonate (Figs. 3a, 3b). Chalcopyrite emulsion inclusions in sphalerite coincides to chalcopyrite-galena intergrowths that form chains and indicates replacement of sphalerite with these minerals (Fig. 2g). Probably, there are two fahlore generations at the deposit: the earlier one is represented by tetrahedrite that forms mutual intergrowths with chalcopyrite, galena, and sphalerite; the later one overgrows the earlier tetrahedrite aggregates, replaces chalcopyrite, and can be found within sphalerite and galena in the thin veinlets.

Such features as the similar intergrowths of tetrahedrite and sphalerite relative to early minerals, smooth boundaries, absence of intercepts and corrosion interactions between them indicate the close formation time of these minerals when equilibrium conditions were attained.

\section{CHEMICAL COMPOSITION OF TETRAHEDRITE AND SPHALERITE}

The chemical composition of tetrahedrite and sphalerite was study with a Camebax Microbeam electron microprobe (EMPA) (analyst N.N. Kononkova, Vernadsky Institute of Geochemistry and Analytical Chemistry, Russian Academy of Sciences, Moscow). The analytical conditions were as follows: measuring current, $30 \mathrm{nA}$; accelerating voltage, $20 \mathrm{kV}$; beam diameter, $2-5 \mu \mathrm{m}$.

According to a study by electron microprobe analysis (EMPA) (51 analyses), tetrahedrite-( $\mathrm{Zn})$ from the Teremki deposit contains low concentrations of $\mathrm{Ag}$ (0.5-0.9 wt \%) and $\mathrm{Bi}$ (up to $1 \mathrm{wt} \%)$ : the $\mathrm{Sb} /(\mathrm{Sb}+$ As) ratio is $0.66-0.97$, and the $\mathrm{Fe} /(\mathrm{Fe}+\mathrm{Zn})$ ratio is 0.27-0.43 (Fig. 4a). In tetrahedrite, the mentioned ratios show a medium negative correlation (the correlation coefficient is -0.6 ).

According to 15 EPMA, sphalerite from the deposit contains low Fe contents, $0.52-2.39$ wt \% (FeS content, $0.9-4.2 \mathrm{~mol} \%$ ); the concentrations of $\mathrm{Cu}$ and Cd do not exceed 0.56 and $0.24-0.86$ wt \%, respectively. It was reported earlier that the Fe concentration in sphalerite varied from 1.74 to $8.2 \mathrm{wt} \%$, corresponding to an FeS content of 3.0-13.8 mol \% (Prokof'ev et al., 2004).

Coexisting tetrahedrite-( $\mathrm{Zn})$ and sphalerite were found in a sample from vein no. 2 (sample 2419sht82). The composition of tetrahedrite-( $\mathrm{Zn})$ is as follows (wt \%): 38.76-39.86 Cu, 0.60-0.94 Ag, 4.69-5.54 Zn, 1.82$2.63 \mathrm{Fe}, 0.11-0.27 \mathrm{Sn}$, up to $0.14 \mathrm{Cd}$, up to $0.17 \mathrm{Hg}$, 19.96-27.95 $\mathrm{Sb}, 0.50-6.46 \mathrm{As}, 0.05-0.77 \mathrm{Bi}$, up to $0.07 \mathrm{Te}, 24.23-24.86 \mathrm{~S}$, and up to $0.09 \mathrm{Se}$; the generalized formula is $\left(\mathrm{Cu}_{10.09-10.24} \mathrm{Ag}_{0.09-0.14}\right)_{\Sigma(10.20-10.38)}$ $\left(\mathrm{Zn}_{1.18-1.40} \mathrm{Fe}_{0.54-0.78} \mathrm{Sn}_{0.02-0.04} \mathrm{Cd}_{0-0.02} \mathrm{Hg}_{0-0.01}\right)_{\Sigma(1.95-2.02)}$ $\left(\mathrm{Sb}_{2.68-3.83} \mathrm{As}_{0.11-1.41} \mathrm{Bi}_{0-0.06} \mathrm{Te}_{0-0.01}\right)_{\Sigma(3.96-4.17)}\left(\mathrm{S}_{12.53-12.70}\right.$ $\left.\mathrm{Se}_{0-0.02}\right)_{\Sigma(12.53-12.70)}$; the $\mathrm{Sb} /(\mathrm{Sb}+\mathrm{As})$ and $\mathrm{Fe} /(\mathrm{Fe}+$ $\mathrm{Zn}$ ) ratios are $0.66-0.97$ and $0.28-0.40$, respectively (Tables 1,2). The composition of sphalerite is as follows (wt \%): 64.36-65.51 Zn, 0.88-1.43 Fe, up to $0.56 \mathrm{Cu}, 0.29-0.86 \mathrm{Cd}$, and $32.64-33.42 \mathrm{~S}$; the gen-

\footnotetext{
Fig. 1. $(a, b)$ Schematic geographic location and (c) schematic geological setting of Teremki gold-ore deposit (East Transbaikalia). Geological scheme is compiled on basis of material collected by Darasun geological survey expedition and with use of geological survey on scale of $1: 200$ 000. (1) Metamorphic complex $\left(\mathrm{PR}_{1}\right)$ represented by gneisses and crystalline schists; (2) Kruchininskii gabbro-amphibolite complex $\left(\mathrm{Pz}_{1} k\right)$; (3-6) Krestovskii Complex $\left(\mathrm{Pz}_{1} k r\right)$ : (3) granodiorites and diorites, (4) granites and plagiogranites, (5) granites, (6) lamprophyre dikes; $(7,8)$ Amanskii Complex $(\mathrm{T} a)$ : (7) granites, granodiorites, diorites, and monzodiorites, (8) granites and alaskites; (9-13) Amudzhikan Complex $\left(\mathrm{J}_{3} a s\right)$ : (9) rhyolites, felsites, and their tuffs and lava breccias, (10) granite porphyries and plagiogranite porphyries, (11) dikes of granite porphyries and plagiogranite porphyries, (12) felsite dikes, (13) lamprophyre dikes; (14) Nerchugan Complex of alkaline granite $\left(\mathrm{J}_{3} n\right) ;(15,16)$ Early Cretaceous subvolcanic complex $\left(\mathrm{K}_{1}\right)$ : (15) rhyolites, felsites, perlites, and obsidian, (16) felsite dikes; (17) alluvial sediments $\left(\mathrm{Q}_{\mathrm{IV}}\right)$; (18) tectonic faults: (a) proven, (b) inferred; (19) out-of-scale metasomatic bodies of (a) beresites and (b) tourmalinites; (20) deposit contour.
} 

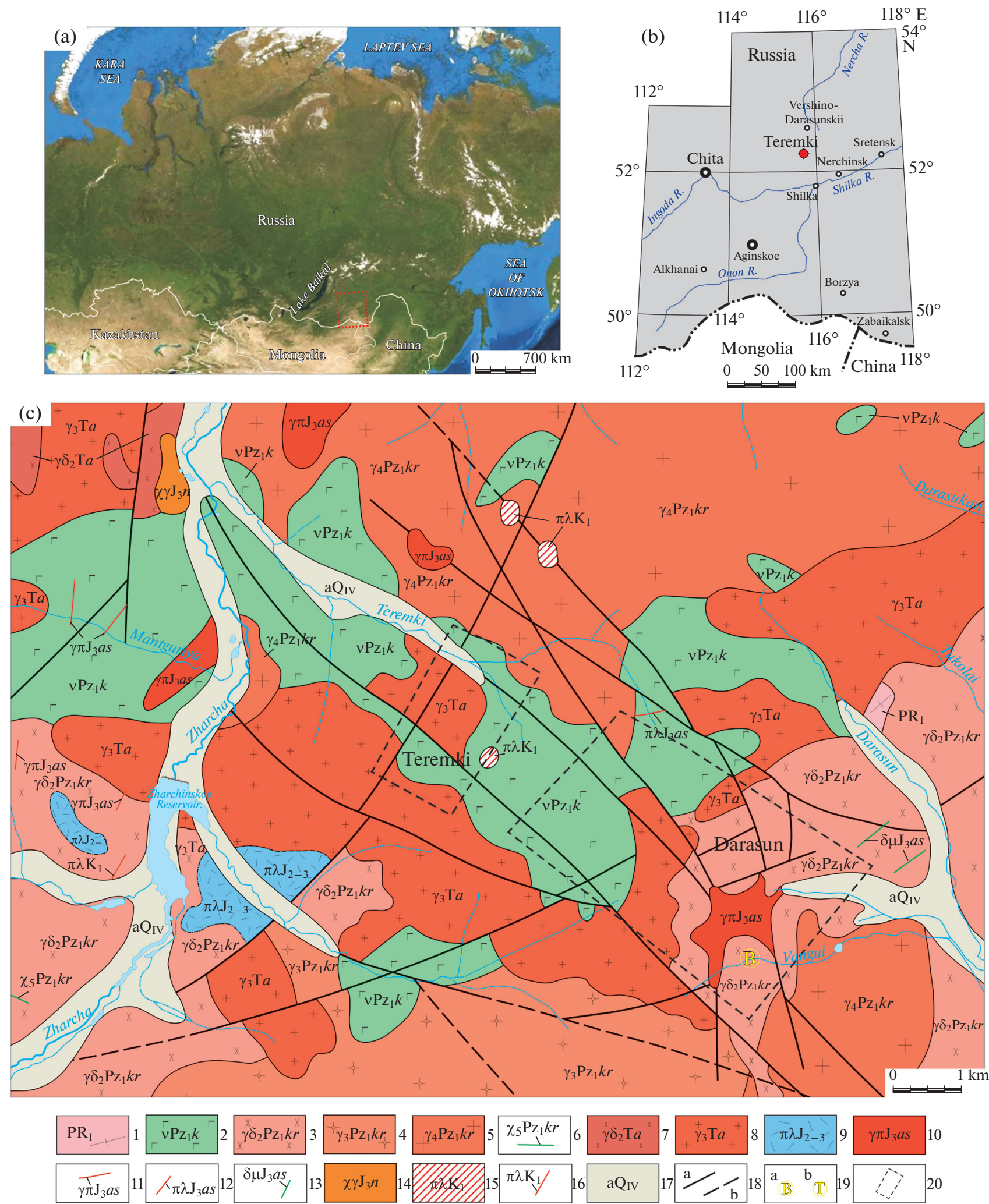

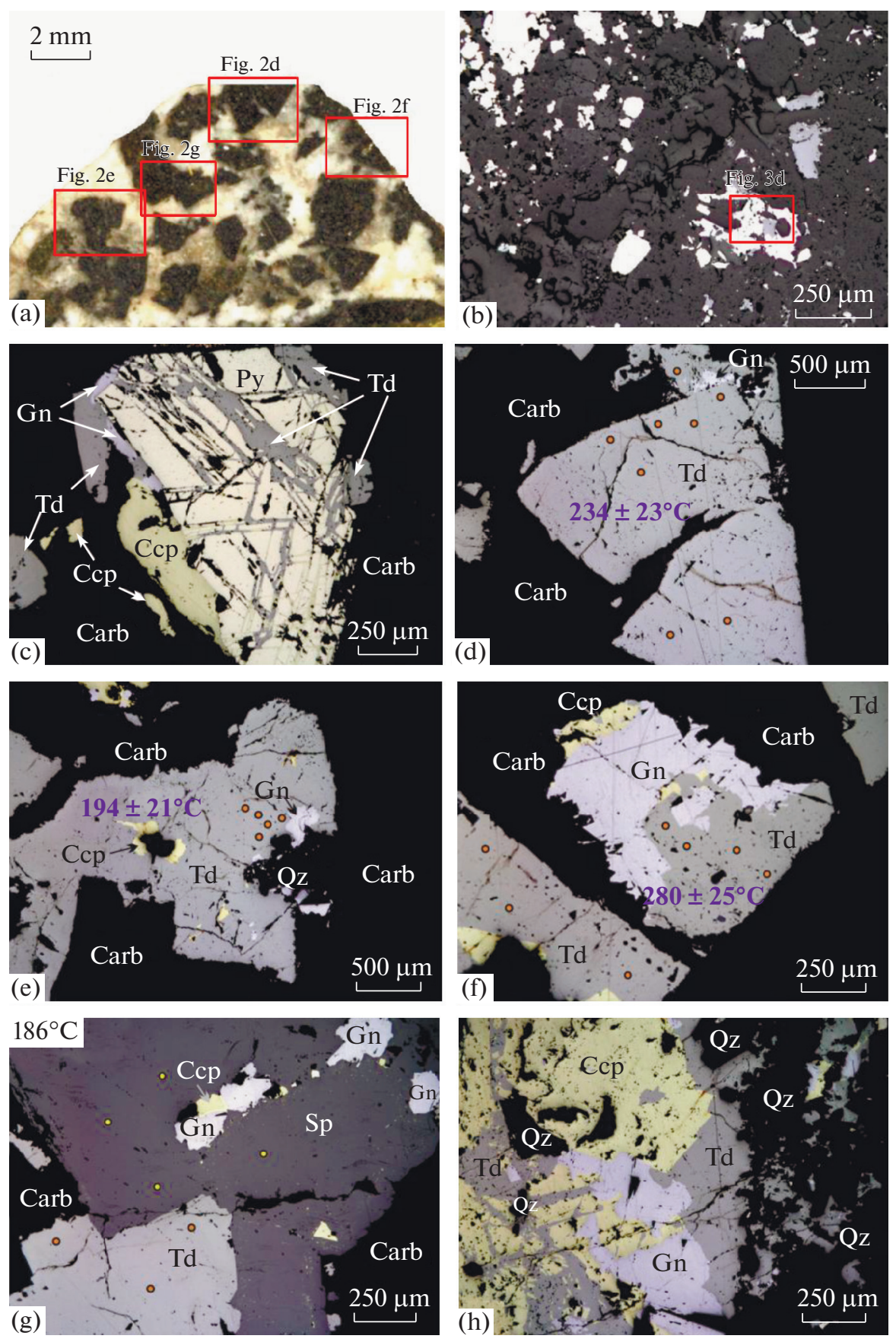

Fig. 2. Mineral relationships in ores of Teremki deposit. (a) Fragments of sulfides and their intergrowths (black) cemented with carbonate (white). (b) Sulfide aggregates forming nodules and impregnation (white) in hosting gangue minerals (dark gray); together with carbonate (Carb), sulfides fill interstices between quartz grains (Qz). (c) Cataclastic pyrite (Py) cemented with galena-chalcopyrite-tetrahedrite aggregate. (d) Galena-tetrahedrite aggregate grows over coarse-grained tetrahedrite (Td), cracks in aggregate are filled with carbonate. (e) Tetrahedrite aggregate with inclusions of chalcopyrite (Ccp) and galena (Gn), hosted in carbonate. (f) Grains of tetrahedrite and tetrahedrite intergrown with galena and chalcopyrite, cemented with carbonate. (g) Intergrowth of tetrahedrite, sphalerite $(\mathrm{Sp})$, and galena; galena intergrown with chalcopyrite forms chained segregations in sphalerite; at contacts with sulfides and in periphery, sphalerite contains emulsion inclusions of chalcopyrite. (h) Galena-chalcopyrite-tetrahedrite aggregate filling interstices between quartz grains; tetrahedrite forms mutual smooth-boundary intergrowths with chalcopyrite and also replaces chalcopyrite to form a network of veinlets. Hereinafter, points mark EMPA analyses with denoted temperatures calculated for coexisting tetrahedrite-( $\mathrm{Zn})$ and sphalerite (on averaged compositions) and for inhomogeneous tetrahedrite-(Zn). Photomicrographs are under reflected light. 

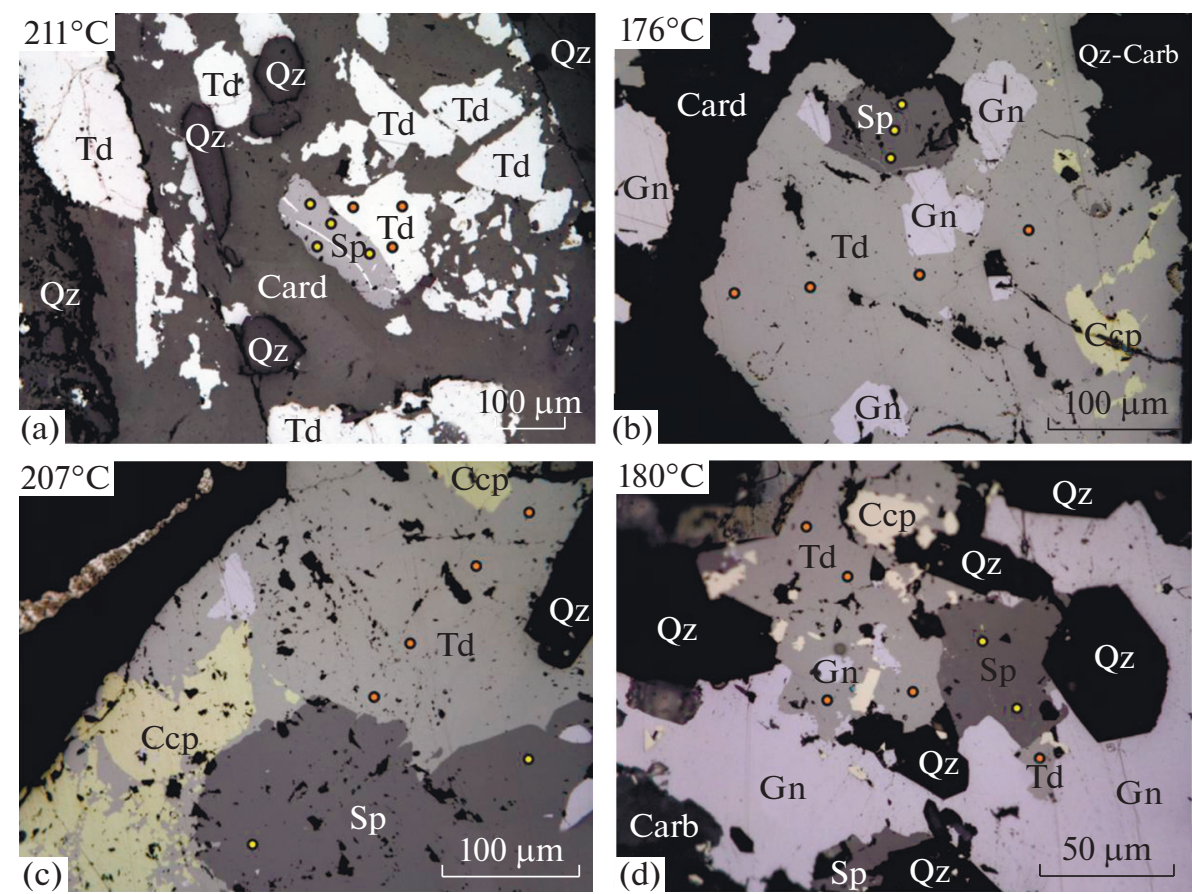

Fig. 3. Relationships of coexisting tetrahedrite-( $\mathrm{Zn})$ and sphalerite in ores of Teremki deposit. (a) Fragments of grains of tetrahedrite and tetrahedrite intergrown with sphalerite; crystals of quartz are cemented with carbonate. (b) Tetrahedrite aggregate with inclusions of sphalerite, galena, and chalcopyrite, hosted in carbonate. (c) Anhedral intergrowth of tetrahedrite, sphalerite, and chalcopyrite; tetrahedrite replaces chalcopyrite to form veinlets in it and corrosion marks on rims, penetrates along contact between chalcopyrite and sphalerite, and contains galena inclusions. (d) Sphalerite-galena-chalcopyrite-tetrahedrite aggregate with carbonate filling interstices between quartz grains. Photomicrographs are under reflected light.

(a)

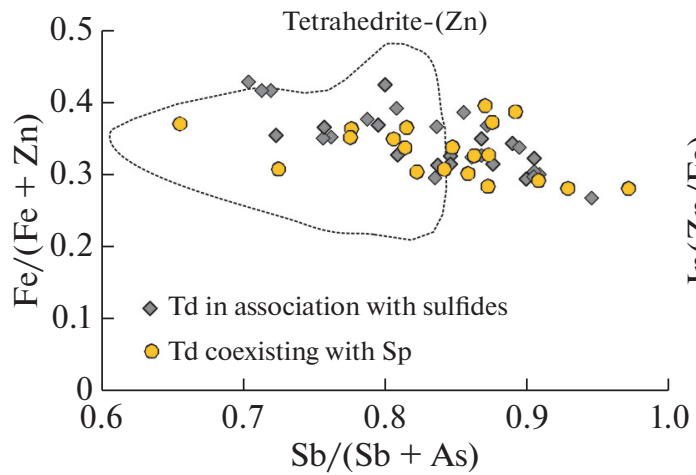

(b)

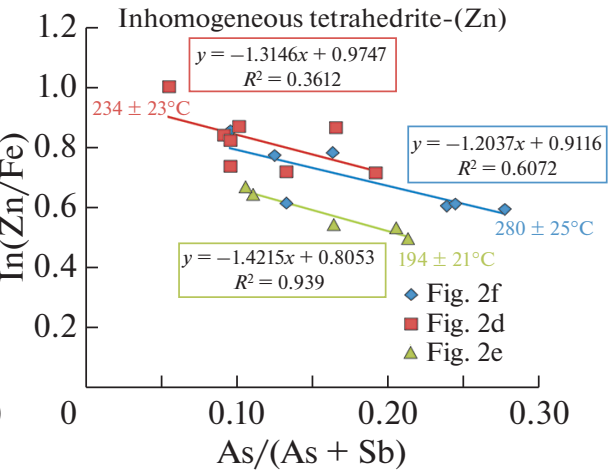

(c)

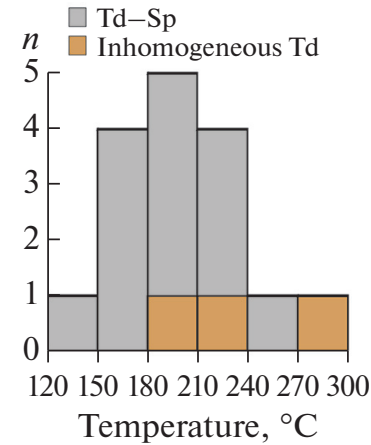

Fig. 4. Composition of $(\mathrm{a}, \mathrm{b})$ tetrahedrite- $(\mathrm{Zn})$ and $(\mathrm{c})$ frequency distribution of calculated crystallization temperatures of tetrahedrite-sphalerite paragenesis from Teremki deposit. Dashed line in Fig. 4a denotes compositional domain of fahlore coexisting with sphalerite from Darasun deposit (Lyubimtseva et al., 2018b). $n$-frequency.

eralized formula is $\left(\mathrm{Zn}_{0.96-0.97} \mathrm{Fe}_{0.02-0.03} \mathrm{Cu}_{0-0.01} \mathrm{Cd}_{0-}\right.$ $\left.{ }_{0.01}\right)_{\Sigma(0.99-1.00)} \mathrm{S}_{1.00-1.01}$; the FeS content is $1.5-2.5 \mathrm{~mol} \%$.

\section{FORMATION CONDITIONS OF SPHALERITE- TETRAHEDRITE PARAGENESES}

The aforementioned relationships between tetrahedrite and sphalerite suppose equilibrium crystalliza- tion of these minerals, therefore, this pair of minerals can be used to determine formation temperature using geothermomentric methods.

\section{Crystallization Temperatures of Coexisting Tetrahedrite-(Zn) and Sphalerite}

Crystallization temperatures of tetrahedrite and sphalerite were determined using (1) the geother- 


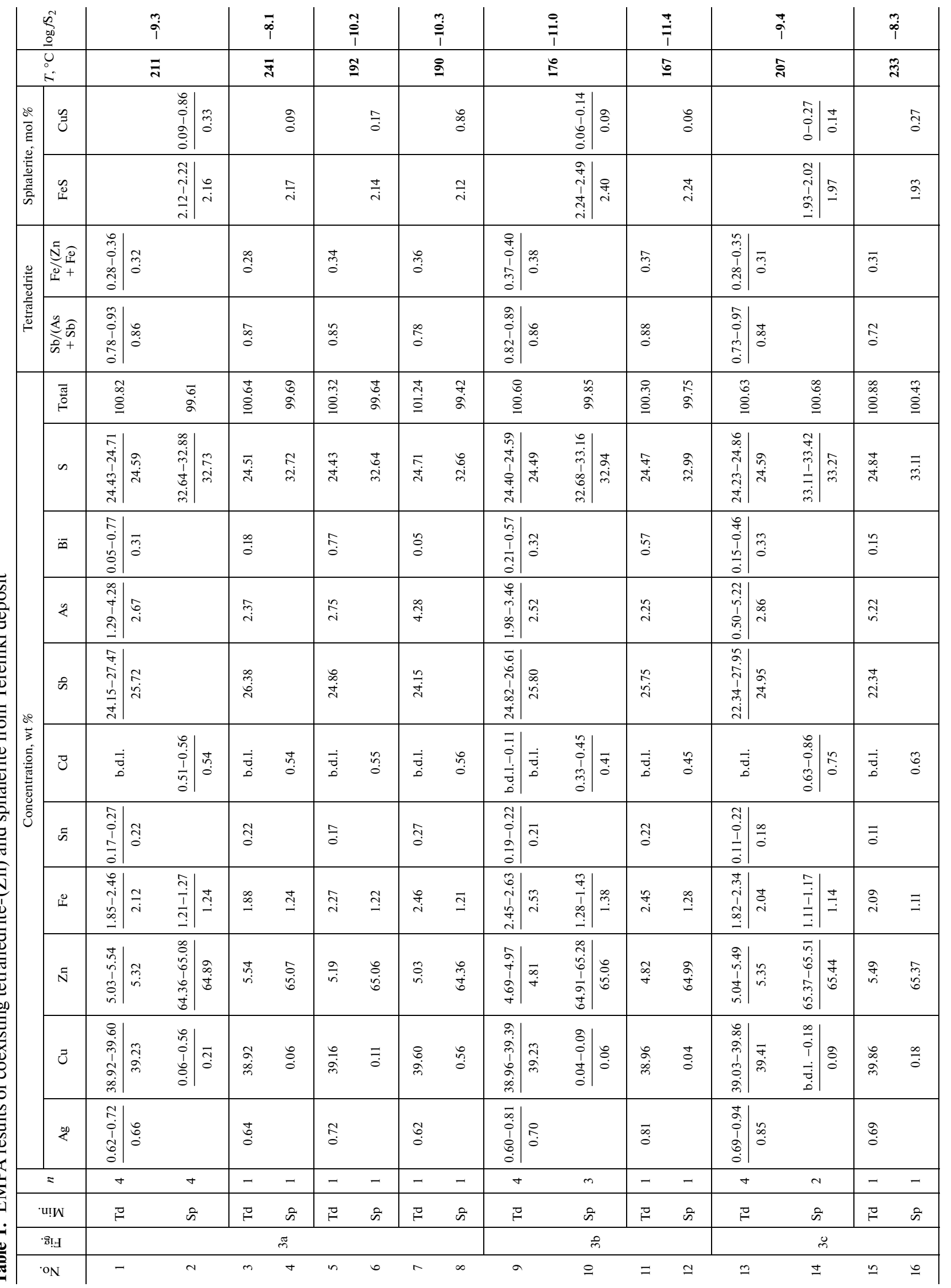




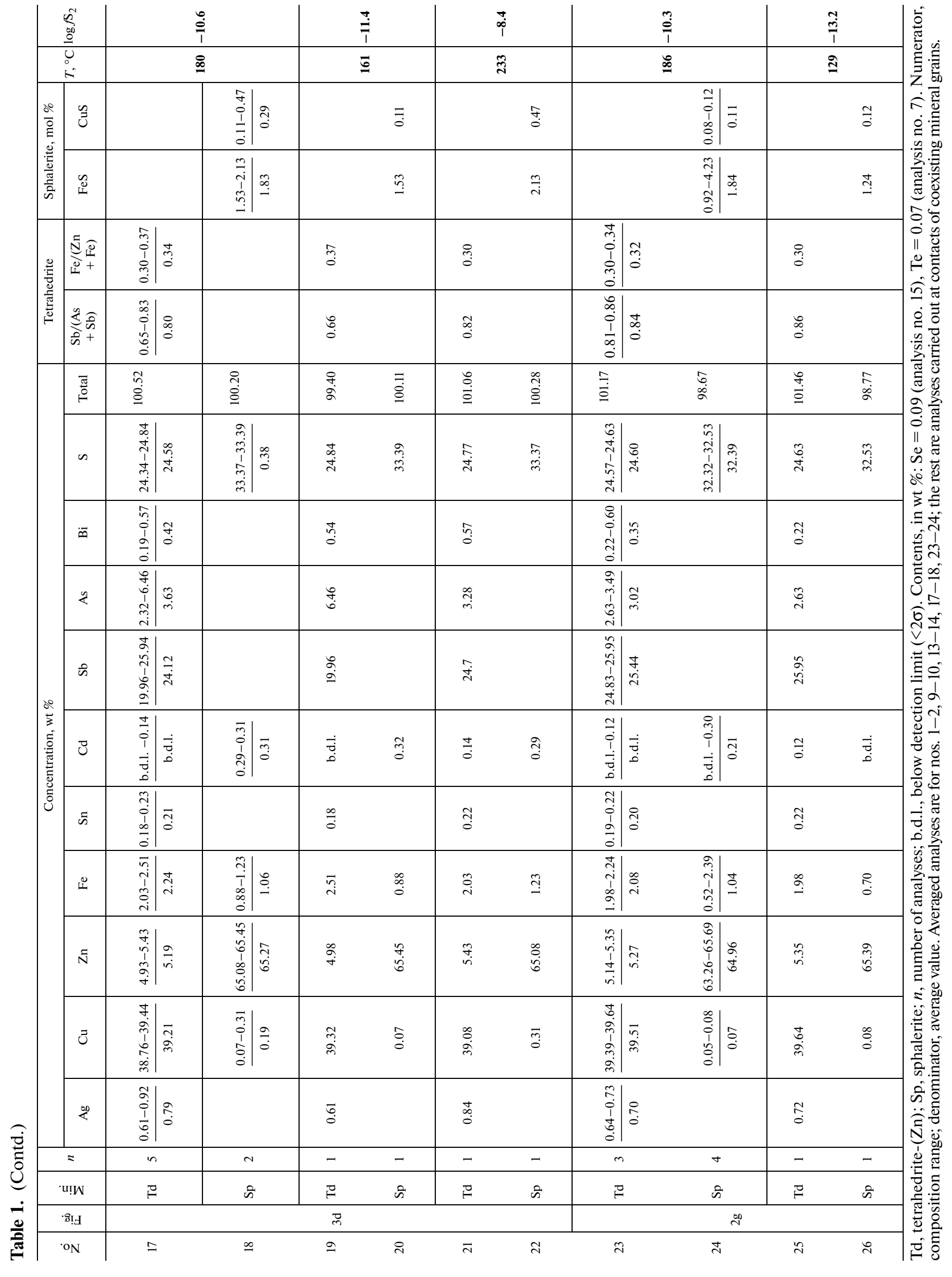




\begin{tabular}{|c|c|c|c|c|c|c|c|c|c|c|c|c|c|c|c|c|c|c|c|c|c|c|}
\hline & in & & & & 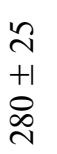 & & & & \multicolumn{8}{|c|}{$\begin{array}{l}\tilde{N} \\
+1 \\
+ \\
\tilde{N}\end{array}$} & \multicolumn{5}{|c|}{$\begin{array}{l}\vec{\sim} \\
+1 \\
\dot{\sigma}\end{array}$} & \\
\hline \multicolumn{2}{|c|}{$\Sigma$} & & & & 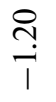 & & & & \multicolumn{8}{|c|}{$\vec{\oplus}$} & \multicolumn{5}{|c|}{$\underset{I}{\stackrel{\overbrace{}}{i}}$} & \\
\hline \multicolumn{2}{|c|}{$\begin{array}{l}\widehat{0} \\
\stackrel{\Xi}{ \pm} \\
\Xi\end{array}$} & $\begin{array}{l}\infty \\
\infty \\
0\end{array}$ & $\stackrel{\vec{\sigma}}{0}$ & $\stackrel{\infty}{\stackrel{0}{0}}$ & $\frac{\infty}{\stackrel{0}{0}}$ & $\begin{array}{l}\stackrel{8}{0} \\
\stackrel{0}{0}\end{array}$ & గ్ర & $\begin{array}{l}\vec{\sigma} \\
0\end{array}$ & $\begin{array}{l}+ \\
\infty \\
0\end{array}$ & $\stackrel{\mathbb{2}}{\mathfrak{0}}$ & $\begin{array}{l}\text { OD } \\
0 \\
0\end{array}$ & $\begin{array}{c}\hat{\infty} \\
0 \\
0\end{array}$ & $\stackrel{8}{\stackrel{-}{-}}$ & $\begin{array}{l}\hat{\infty} \\
0 \\
0\end{array}$ & $\stackrel{\mathbb{2}}{\stackrel{0}{0}}$ & $\stackrel{+}{\stackrel{0}{0}}$ & $\tilde{n}$ & $\stackrel{n}{6}$ & ڤ్ర & ?n & sa & \\
\hline \multicolumn{2}{|c|}{$\begin{array}{l}\hat{a} \\
n \\
+ \\
\frac{1}{3} \\
\frac{n}{4}\end{array}$} & $\stackrel{0}{\stackrel{0}{0}}$ & $\underset{\sim}{\stackrel{*}{0}}$ & $\stackrel{0}{0}$ & $\stackrel{7}{0}$ & $\underset{\sim}{\infty}$ & $\frac{m}{0}$ & 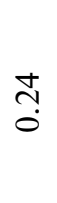 & $\stackrel{\text { oे }}{0}$ & $\stackrel{m}{0}$ & $\begin{array}{l}\stackrel{a}{0} \\
\stackrel{0}{0}\end{array}$ & $\stackrel{\circ}{0}$ & $\stackrel{2}{0}$ & $\frac{0}{0}$ & $\frac{9}{0}$ & $\stackrel{\partial}{0}$ & $\stackrel{\widetilde{\jmath}}{0}$ & $=$ & $=$ & $\frac{0}{0}$ & $\bar{\jmath}$ & \\
\hline \multicolumn{2}{|c|}{ 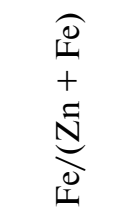 } & ஸे & $\ddot{n}$ & $\overrightarrow{\tilde{o}}$ & $\stackrel{\sim}{0}$ & లి & $\tilde{?}$ & $\stackrel{n}{\tilde{c}}$ & ஸे & $\stackrel{m}{m}$ & ஸे & సે & $\grave{\hat{o}}$ & ڤ్లి & $\stackrel{n}{n}$ & กె & $\hat{n}$ & $\stackrel{+}{m}$ & $\stackrel{+}{n}$ & $\hat{n}$ & $\stackrel{\infty}{\tilde{a}}$ & \\
\hline \multirow{10}{*}{ 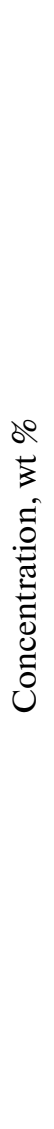 } & 营 & 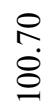 & $\stackrel{\substack{+\dot{\sigma}}}{ }$ & $\begin{array}{l}\overline{6} \\
\dot{8}\end{array}$ & 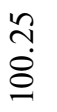 & $\begin{array}{l}\stackrel{+}{\infty} \\
\dot{0}\end{array}$ & $\begin{array}{l}\text { ণิ } \\
\stackrel{8}{8}\end{array}$ & $\begin{array}{l}\hat{\infty} \\
\dot{\delta}\end{array}$ & $\begin{array}{l}\overrightarrow{0} \\
\dot{8}\end{array}$ & $\begin{array}{l}\stackrel{N}{8} \\
\stackrel{8}{O}\end{array}$ & 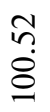 & $\begin{array}{l}\stackrel{2}{2} \\
\stackrel{8}{8}\end{array}$ & $\begin{array}{l}\dot{\sigma} \\
\text { aे }\end{array}$ & $\stackrel{\widehat{\hat{\sigma}}}{\stackrel{0}{0}}$ & ஸे & $\begin{array}{l}\stackrel{8}{0} \\
\stackrel{8}{8}\end{array}$ & 옹 & ণิ & $\begin{array}{l}\hat{\sigma} \\
\grave{8}\end{array}$ & $\stackrel{m}{\stackrel{3}{0}}$ & in & \\
\hline & $n$ & $\begin{array}{l}\text { ๗े } \\
\stackrel{\sim}{\sim}\end{array}$ & $\begin{array}{l}\text { よ̆ } \\
\dot{j}\end{array}$ & $\begin{array}{l}\stackrel{+}{\sim} \\
\stackrel{\sim}{\sim}\end{array}$ & $\stackrel{\infty}{\underset{+}{\sim}}$ & $\begin{array}{l}= \\
\ddot{n}\end{array}$ & $\begin{array}{l}\stackrel{\sim}{+} \\
\stackrel{\sim}{\sim}\end{array}$ & $\begin{array}{l}\stackrel{શ}{\Delta} \\
\stackrel{\sim}{N}\end{array}$ & $\begin{array}{l}\stackrel{n}{\sim} \\
\stackrel{+}{\sim}\end{array}$ & $\begin{array}{l}\text { f. } \\
\stackrel{+}{\sim}\end{array}$ & $\begin{array}{l}\text { ले } \\
\stackrel{\sim}{\sim}\end{array}$ & $\begin{array}{l}\stackrel{+}{+} \\
\stackrel{+}{\sim}\end{array}$ & $\begin{array}{l}\text { बे } \\
\text { ते }\end{array}$ & $\begin{array}{l}\hat{\sigma} \\
\dot{\sim}\end{array}$ & $\begin{array}{l}\infty \\
\infty \\
\dot{\sim}\end{array}$ & $\begin{array}{l}\stackrel{?}{+} \\
\stackrel{\sim}{\sim}\end{array}$ & $\begin{array}{l}\tilde{\sigma} \\
\dot{\sim}\end{array}$ & $\begin{array}{l}\hat{n} \\
\stackrel{d}{d}\end{array}$ & $\begin{array}{l}\stackrel{\sim}{\sim} \\
\stackrel{+}{\sim}\end{array}$ & $\begin{array}{l}+ \\
\dot{d} \\
\dot{\sim}\end{array}$ & a & \\
\hline & $\bar{n}$ & $\stackrel{m}{\tilde{0}}$ & $\frac{\infty}{0}$ & فِّه & $\stackrel{n}{\stackrel{n}{0}}$ & $\underset{\sim}{\infty}$ & $\stackrel{\stackrel{\sim}{\leftarrow}}{\circ}$ & $\tilde{n}$ & $\stackrel{n}{n}$ & ָิ & $\stackrel{\vec{\sigma}}{0}$ & $\begin{array}{l}8 \\
:\end{array}$ & $\stackrel{i}{0}$ & Oे. & $\tilde{\widetilde{o}}$ & $\stackrel{m}{0}$ & $\stackrel{\overbrace{}}{0}$ & กิ & $\begin{array}{l}\vec{\infty} \\
0\end{array}$ & n̊. & ले & \\
\hline & $\frac{2}{4}$ & $\stackrel{\mathbb{S}}{\stackrel{S}{-}}$ & $\stackrel{\sim}{\sim}$ & $\begin{array}{l}n \\
\text { rn. }\end{array}$ & $\stackrel{\sim}{\sim}$ & $\stackrel{\vec{m}}{i}$ & $\underset{i}{\vec{J}}$ & $\begin{array}{l}\stackrel{\imath}{r} \\
\stackrel{r}{*}\end{array}$ & $\underset{-}{\sigma}$ & $\stackrel{\nabla}{i}$ & $\stackrel{\text { oे }}{.}$ & Sֵ & $\stackrel{0}{\circ}$ & $\stackrel{\circ}{\stackrel{ }{m}}$ & $\begin{array}{l}\tilde{r} \\
\dot{r}\end{array}$ & $\stackrel{+}{\stackrel{.}{~}}$ & $\begin{array}{l}\text { के } \\
\text { ஸे }\end{array}$ & $\begin{array}{l}\text { ô } \\
\text { in }\end{array}$ & $\stackrel{8}{\circ}$ & 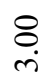 & হ & \\
\hline & ஜ & $\begin{array}{l}\bar{\sigma} \\
\dot{\sigma}\end{array}$ & 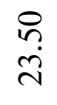 & $\begin{array}{l}\tilde{n} \\
\tilde{n}\end{array}$ & $\begin{array}{l}\infty \\
\infty \\
\ddot{\lambda}\end{array}$ & $\begin{array}{l}\stackrel{0}{0} \\
\underset{\sim}{ }\end{array}$ & $\begin{array}{l}\stackrel{R}{2} \\
\ddot{\sim}\end{array}$ & $\begin{array}{l}\vec{\sigma} \\
\ddot{\sim}\end{array}$ & 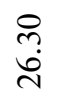 & 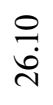 & $\begin{array}{l}\overrightarrow{\text { }} \\
\text {. }\end{array}$ & 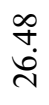 & 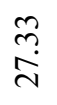 & $\begin{array}{l}\tilde{n} \\
\ddot{n} \\
\ddot{n}\end{array}$ & $\begin{array}{l}\hat{\infty} \\
\dot{\sim} \\
\dot{N}\end{array}$ & 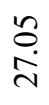 & $\begin{array}{l}\stackrel{י}{\sim} \\
\stackrel{d}{\sim}\end{array}$ & $\begin{array}{l}\text { bे } \\
\text { ढे }\end{array}$ & 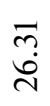 & $\begin{array}{l}\stackrel{\circ}{+} \\
\dot{+}\end{array}$ & $\stackrel{\Delta}{\nearrow}$ & \\
\hline & $\tilde{\tilde{n}}$ & $\frac{9}{0}$ & $\stackrel{\overbrace{}}{0}$ & กิ & సి & $\begin{array}{l}0 \\
0\end{array}$ & $\stackrel{m}{0}$ & $\stackrel{\text { กิ }}{0}$ & $\frac{9}{0}$ & $\stackrel{n}{0}$ & 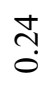 & ָָ & స̃ & $\frac{9}{0}$ & సి & 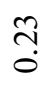 & Э. & $\stackrel{m}{0}$ & $\stackrel{\infty}{0}$ & $\frac{9}{0}$ & $\tilde{\tilde{a}}$ & \\
\hline & 蒫 & $\stackrel{n}{g}$ & $\stackrel{\sim}{\sim}$ & $\begin{array}{l}\stackrel{0}{0} \\
\text { i }\end{array}$ & $\begin{array}{l}\text { ñ } \\
\text { in }\end{array}$ & ले & तે & $\stackrel{m}{i}$ & 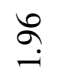 & $\stackrel{n}{i}$ & $\begin{array}{l}\stackrel{8}{0} \\
\text { i }\end{array}$ & $\widehat{\Omega}$ & $\stackrel{n}{\stackrel{n}{-}}$ & $\stackrel{\infty}{\stackrel{2}{-}}$ & $\stackrel{\vec{c}}{i}$ & $\underset{\sim}{\exists}$ & $\stackrel{\infty}{\stackrel{\infty}{\sim}}$ & $\underset{\sim}{\stackrel{\sim}{N}}$ & 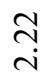 & $\underset{\sim}{\vec{f}}$ & is & \\
\hline & Nี & $\begin{array}{l}\infty \\
\stackrel{m}{n}\end{array}$ & $\begin{array}{l}\stackrel{n}{o} \\
\ddot{n}\end{array}$ & $\stackrel{\infty}{\stackrel{\infty}{n}}$ & $\underset{\sim}{i n}$ & $\begin{array}{l}\infty \\
0 \\
\dot{n}\end{array}$ & $\stackrel{\circ}{\stackrel{+}{+}}$ & $\begin{array}{l}\Xi_{0} \\
\text { in }\end{array}$ & $\stackrel{m}{n}$ & $\stackrel{5}{n}$ & $\begin{array}{l}\dot{m} \\
\dot{n}\end{array}$ & $\begin{array}{l}\infty \\
\text { nn. } \\
\text { n. }\end{array}$ & $\begin{array}{l}\text { ñ } \\
\text { in }\end{array}$ & $\begin{array}{l}\vec{n} \\
i n\end{array}$ & $\begin{array}{l}\text { ते } \\
\text { in }\end{array}$ & $\frac{5}{n}$ & $\stackrel{n}{\sigma}$ & $\begin{array}{l}\hat{\theta} \\
\dot{n}\end{array}$ & $\begin{array}{l}\infty \\
0 \\
\dot{n}\end{array}$ & $\begin{array}{r}\infty \\
\dot{\sigma}\end{array}$ & $\delta$ & \\
\hline & $\tilde{J}$ & $\begin{array}{l}\stackrel{+}{*} \\
\text { ळे }\end{array}$ & $\begin{array}{l}\stackrel{\infty}{\circ} \\
\text { ळे }\end{array}$ & $\begin{array}{l}\tilde{n} \\
\text { ले }\end{array}$ & $\begin{array}{l}f \\
\text { iे }\end{array}$ & $\frac{\dot{t}}{\dot{q}}$ & $\begin{array}{l}\text { ị } \\
\text { ले }\end{array}$ & $\begin{array}{l}\stackrel{P}{\circ} \\
\text { ळे }\end{array}$ & $\begin{array}{l}0 \\
\infty \\
\infty \\
\infty\end{array}$ & $\begin{array}{l}\text { ஸे } \\
\text { ले }\end{array}$ & $\begin{array}{l}\text { ते } \\
\text { ले }\end{array}$ & $\begin{array}{l}\stackrel{ \pm}{n} \\
\text { mे }\end{array}$ & $\begin{array}{l}\bar{\sigma} \\
\infty \\
\infty\end{array}$ & $\begin{array}{l}\stackrel{0}{0} \\
\stackrel{0}{ }\end{array}$ & $\begin{array}{l}3 \\
\text { 乃े } \\
\text { in }\end{array}$ & $\begin{array}{l}\text { ô. } \\
\text { ळे }\end{array}$ & $\begin{array}{l}\bar{a} \\
\text { ळे }\end{array}$ & $\begin{array}{l}\text { రె } \\
\text { ळे}\end{array}$ & $\begin{array}{l}\text { fे. } \\
\stackrel{\text { mे }}{ }\end{array}$ & $\begin{array}{l}\tilde{n} \\
\stackrel{n}{ }\end{array}$ & $\begin{array}{l}\text { to } \\
\text { ले }\end{array}$ & \\
\hline & $\frac{00}{2}$ & $\stackrel{0}{0}$ & $\stackrel{3}{0}$ & \begin{tabular}{l}
\multirow{H}{*}{} \\
$\stackrel{0}{0}$
\end{tabular} & ñn & $\stackrel{\hat{\imath}}{0}$ & $\stackrel{n}{0}$ & $\underset{\stackrel{\infty}{0}}{0}$ & $\stackrel{n}{n}$ & $\begin{array}{l}n \\
0 \\
0\end{array}$ & \begin{tabular}{l}
\multirow{H}{*}{} \\
$\stackrel{0}{0}$
\end{tabular} & $\begin{array}{l}\text { : } \\
\stackrel{0}{0}\end{array}$ & $\stackrel{\infty}{+}$ & $\tilde{n}$ & $\begin{array}{l}\vec{b} \\
0\end{array}$ & $\stackrel{\mathbb{2}}{\stackrel{0}{0}}$ & $\begin{array}{l}\infty \\
\stackrel{0}{0} \\
0\end{array}$ & $\begin{array}{l}0 \\
\stackrel{0}{0}\end{array}$ & $\stackrel{\mathbb{2}}{\stackrel{0}{0}}$ & $\vec{i}$ & $\stackrel{\infty}{2}$ & \\
\hline \multicolumn{2}{|c|}{$=$} & \multicolumn{7}{|c|}{$r$} & \multicolumn{8}{|c|}{$\infty$} & \multicolumn{4}{|c|}{$n$} & & \\
\hline \multicolumn{2}{|c|}{$\stackrel{\infty 00}{\dot{D}}$} & \multicolumn{7}{|c|}{$\overleftrightarrow{\sim}$} & \multicolumn{8}{|c|}{$\vec{\sim}$} & \multicolumn{5}{|c|}{$\stackrel{\sim}{\sim}$} & \\
\hline & $\dot{\mathrm{z}}$ & - & $\sim$ & $m$ & $\sigma$ & in & 6 & $r$ & $\infty$ & $a$ & 으 & $=$ & $\simeq$ & $\underline{n}$ & $\Xi$ & $\bumpeq$ & 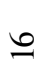 & $=$ & $\stackrel{\infty}{.}$ & 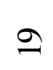 & & \\
\hline
\end{tabular}


mometer from (Sack and Loucks, 1985), which is based on the data on distribution of $\mathrm{Fe}$ and $\mathrm{Zn}$ between these minerals, and (2) the thermodynamic model of tennantite-tetrahedrite solid solution. Distribution of $\mathrm{Fe}$ and $\mathrm{Zn}$ between coexisting fahlore and sphalerite is characterized by the exchange reaction of $\mathrm{Fe}$ and $\mathrm{Zn}$ between tetrahedrite and sphalerite,

$$
1 / 2 \mathrm{Cu}_{10} \mathrm{Fe}_{2} \mathrm{Sb}_{4} \mathrm{~S}_{13}+\underset{\mathrm{Sp}}{\mathrm{ZnS}} \mathrm{Zn}=1 / 2 \mathrm{Cu}_{10} \underset{\mathrm{Td}-(\mathrm{Zn})}{\mathrm{Zn}_{2} \mathrm{Sb}_{4} \mathrm{~S}_{13}}+\underset{\mathrm{Sp}}{\mathrm{FeS}}
$$

and the reciprocal reaction,

$$
\underset{\mathrm{Td}-(\mathrm{Fe})}{\mathrm{Cu}_{10} \mathrm{Fe}_{2} \mathrm{Sb}_{4} \mathrm{~S}_{13}}+\underset{\mathrm{Tn}-(\mathrm{Zn})}{\mathrm{Cu}_{10} \mathrm{Zn}_{2} \mathrm{As}_{4} \mathrm{~S}_{13}}=\underset{\mathrm{Td}-(\mathrm{Zn})}{\mathrm{Cu}_{10} \mathrm{Zn}_{2} \mathrm{Sb}_{4} \mathrm{~S}_{13}}+\underset{\mathrm{Tn}-(\mathrm{Fe})}{\mathrm{Cu}_{10} \mathrm{Fe}_{2} \mathrm{As}_{4} \mathrm{~S}_{13}}
$$

which describe the change of composition in the isomorphous tennantite-tetrahedrite series.

The temperatures calculated from the sphaleritefahlore geothermometer (Sack and Loucks, 1985) range from 130 to $245 \pm 25^{\circ} \mathrm{C}$ and were estimated for five sites shown in Figs. $2 \mathrm{~g}$ and 3. Of the 13 calculated temperature values, eight were obtained from analyses carried out at contacts of coexisting mineral grains $\left(130-245^{\circ} \mathrm{C}\right)$ and five were obtained from averaged analyses of compositions of coexisting mineral grains at every site $\left(175-210^{\circ} \mathrm{C}\right)$ (Table 1$)$.

\section{Crystallization Temperatures of Inhomogeneous Tetrahedrite-( $\mathrm{Zn})$}

Crystallization temperatures of inhomogeneous tetrahedrite-( $\mathrm{Zn})$ coexisting with sphalerite of constant composition were calculated from the geothermometer developed for fahlore grains with oscillatory zoning (Raabe and Sack, 1984). The feasibility of this geothermometer is based on the "local equilibrium principle" introduced by D.S. Korzhinskii (1973). The creators of this geothermometer assume (1) an isothermal deposition of zonal fahlore and (2) a local equilibrium with respect to $\mathrm{Fe}$ and $\mathrm{Zn}$ exchange between fluid and every sequential zone of growth of fahlore; under these conditions, sphalerite maintains a constant composition and $\mathrm{Fe} / \mathrm{Zn}$ ratio in the zone of growth is caused by the conditions of osmotic equilibrium of $\mathrm{Fe}$ and $\mathrm{Zn}$ exchange.

Application of the geothermometer from (Raabe and Sack, 1984) to inhomogeneous fahlore implies that sphalerite, coexisting with this fahlore, is of constant composition. Since the Fe content in sphalerite in its paragenesis with tetrahedrite-( $\mathrm{Zn})$ is almost nonvarying (the range on variation in the FeS content is $1.5-2.5 \mathrm{~mol} \%$ ), we can suggest that an osmotic equilibrium of $\mathrm{Fe}$ and $\mathrm{Zn}$ exchange between tetrahedrite-( $\mathrm{Zn})$ and sphalerite was attained.

Temperature was calculated by the following equation:

$$
T(\mathrm{~K})=-((664 \pm 30) / m),
$$

where $T(\mathrm{~K})$ is temperature in Kelvin; $m$, the slope angle of the curve determined from the points of composition of zonal fahlore, which were placed on the $\mathrm{As} /(\mathrm{As}+\mathrm{Sb})$ versus $\ln (\mathrm{Zn} / \mathrm{Fe})$ plot.
Temperatures for inhomogeneous tetrahedrite$(\mathrm{Zn})$ were calculated for its three area shown in Figs. $2 \mathrm{~d}-2 \mathrm{f}$, and their values range from 195 to $280^{\circ} \mathrm{C}$ (Table 2). Figure $4 \mathrm{~b}$ shows the variations in composition of tetrahedrite-(Zn), with the slope angle determined for a straight regression line for every area.

\section{Sulfur Fugacity}

The sulfur fugacity was estimated from data on the calculated crystallization temperatures of coexisting sphalerite and tetrahedrite-( $\mathrm{Zn})$ and the FeS contents in sphalerite associated with iron sulfides; note that the FeS content is a function of temperature, pressure, and sulfur activity (Barton and Toulmin, 1966; Scott and Barnes, 1971; Dobrovol'skaya et al., 1991). The fugacity was calculated by the equation from (Lusk and Calder, 2004):

$$
\begin{gathered}
\log _{10} f S_{2}=11.01-9.49(1000 / \mathrm{K}) \\
+[0.187-0.252(1000 / \mathrm{K})]\left(\mathrm{FeS}^{\mathrm{Sp}}\right) \\
+[0.35-0.2(1000 / \mathrm{K})]\left(\mathrm{CuS}^{\mathrm{Sp}}\right),
\end{gathered}
$$

where $K$ is the temperature in Kelvin; $\mathrm{FeS}^{\mathrm{Sp}}$ and $\mathrm{CuS}^{\mathrm{Sp}}$ are the molar fractions of $\mathrm{FeS}$ and $\mathrm{CuS}$, respectively, in sphalerite.

The sulfur fugacity values calculated by the equation from (Lusk and Calder, 2004) and corresponding to the conditions when the tetrahedrite-sphalerite paragenesis was deposited fall within the range of $10^{-13.0}-10^{-8.1}$ bars.

\section{DISCUSSION}

It was reported that the ore-forming fluid at the Teremki deposit was close in composition and physicochemical properties to that at the Darasun deposit (Prokof'ev et al., 2004 and references therein), and this is additionally found in the similarity of the mineral compositions in ores. Similarity was also revealed between the fahlore and sphalerite compositions from these two deposits. The iron content of sphalerite from the Teremki deposit varies from 0.52 to $8.20 \mathrm{wt} \%$, corresponding to an FeS content of $0.9-13.8 \mathrm{~mol} \%$ (Prokof'ev et al., 2004 and present work); the same parameters at the Darasun deposit are 0.45-4.71 wt \% and $0.8-8.2 \mathrm{~mol} \%$, respectively (Lyubimtseva et al., $2018 \mathrm{~b}$ and references therein). The fahlore compositions from the two deposits display some differences. 
The Darasun deposit is characterized by the almost complete range of compositions of tennantite-tetrahedrite solid solution with continuous isomorphism between $\mathrm{Sb}$ and As and between Fe and $\mathrm{Zn}$ (Lyubimtseva et al., 2018a). In contrast, only tetrahedrite-(Zn) was found at the Teremki deposit. However, fahlores from both deposits show negative correlations between the $\mathrm{Sb} /(\mathrm{Sb}+\mathrm{As})$ and $\mathrm{Fe} /(\mathrm{Fe}+\mathrm{Zn})$ ratios.

The compositions of coexisting tetrahedrite and sphalerite from these deposits are also similar. The $\mathrm{Fe}$ content in sphalerite from the Teremki deposit is $0.88-1.43$ wt $\%(1.5-2.5 \mathrm{~mol} \% \mathrm{FeS})$, while $0.79-$ 2.80 wt \% (1.4-4.9 mol \% FeS) at the Darasun one (Lyubimtseva et al., 2018b). Tetrahedrite from the Teremki deposit falls within the domain of compositions of tetrahedrite from the Darasun deposit (Fig. 4a), but its Sb content coexisting with sphalerite is higher. $\mathrm{Sb} /(\mathrm{Sb}+\mathrm{As})$ ratios in tetrahedrite from the Teremki deposit changes from 0.66 to 0.97 , while from 0.70 to 0.84 at the Darasun deposit (Lyubimtseva et al., 2018b). $\mathrm{Fe} /(\mathrm{Fe}+\mathrm{Zn})$ ratios in tetrahedrite are similar: $0.28-0.40$ and $0.20-0.48$ at the Teremki and Darasun deposits, respectively (Lyubimtseva et al., 2018b).

The results of the present study show that tetrahedrite-sphalerite paragenesis at the Teremki gold-ore deposit formed at temperatures from 130 to $280^{\circ} \mathrm{C}$ (Fig. 4c) and sulfur fugacity of $10^{-13.0}-10^{-8.1}$ bars. These data are consistent with the homogenization temperatures $\left(216-298^{\circ} \mathrm{C}\right)$ of fluid inclusions in quartz, attributed to the productive stage of the Teremki deposit (Prokof'ev et al., 2004; Prokofiev et al., 2010). Lower temperatures calculated for tetrahedrite-sphalerite aggregates can be explained by a broader crystallization range of tetrahedrite in association with sphalerite and carbonate, but without quartz. The sulfur fugacity calculated in the present study is similar to the fugacity $\left(10^{-15}-10^{-10}\right.$ bars $)$ determined from the homogenization temperatures of inclusions in productive quartz in association with sphalerite (Prokof'ev et al., 2004).

The crystallization temperatures of tetrahedritesphalerite paragenesis from the Teremki deposit (130$280^{\circ} \mathrm{C}$ ) are lower than those at the Darasun deposit $\left(175-355^{\circ} \mathrm{C}\right)$ (Lyubimtseva et al., 2018b); they are more similar to the formation temperatures of the bournonite-seligmanite-fahlore parageneses (100$250^{\circ} \mathrm{C}$ ) of the Darasun deposit (Lyubimtseva et al., 2019). The sulfur fugacity in the formation of the tetrahedrite-sphalerite paragenesis from the Teremki deposit $\left(10^{-13.0}-10^{-8.1}\right.$ bars $)$ is generally close to the same parameter at the Darasun deposit $\left(10^{-11.0}-10^{-5.1}\right.$ bars $)$ (Lyubimtseva et al., 2018b), but its values are lower.

\section{CONCLUSIONS}

(1) The paragenetic association of tetrahedrite$\mathrm{Zn}$ ) and sphalerite has been distinguished and coex- isting compositions of these two minerals from the Teremki gold-ore deposit have been found. The $\mathrm{Sb} /(\mathrm{Sb}+\mathrm{As})$ ratio in tetrahedrite varies from 0.66 to 0.97 ; the $\mathrm{Fe} /(\mathrm{Fe}+\mathrm{Zn})$ ratio, from 0.28 to 0.40 ; a negative correlation has been established between the $\mathrm{Sb} /(\mathrm{Sb}+\mathrm{As})$ and $\mathrm{Fe} /(\mathrm{Fe}+\mathrm{Zn})$ ratios. The Fe content in sphalerite varies from 0.88 to 1.43 wt \% (the $\mathrm{FeS}$ content is $1.5-2.5 \mathrm{~mol} \%$ ).

(2) The formation conditions of the tetrahedritesphalerite paragenesis at the Teremki deposit have been estimated: coexisting tetrahedrite-( $\mathrm{Zn})$ and sphalerite formed at temperatures of $130-280^{\circ} \mathrm{C}$ and a sulfur fugacity of $10^{-13.2}-10^{-8.1}$ bars.

(3) The compositions of minerals (fahlore and sphalerite) and physicochemical parameters of their formation (temperatures and sulfur fugacity) for the studied object and Darasun deposit have been compared. It has been shown that the compositions of fahlore and sphalerite at these deposits are close to each other and the physicochemical parameters of the formation of productive associations of their ore veins are similar.

\section{ACKNOWLEDGMENTS}

The authors are grateful to anonymous reviewers for constructive comments.

\section{FUNDING}

The studies were supported by the state contract no. 121041500220-0 "Structural-Chemical Inhomogeneities and Paragenetic Associations of Minerals as a Reflection of Petrogenesis and Ore Genesis Processes.”

\section{CONFLICT OF INTEREST}

The authors declare that they have no conflict of interest.

\section{OPEN ACCESS}

This article is licensed under a Creative Commons Attribution 4.0 International License, which permits use, sharing, adaptation, distribution and reproduction in any medium or format, as long as you give appropriate credit to the original author(s) and the source, provide a link to the Creative Commons license, and indicate if changes were made. The images or other third party material in this article are included in the article's Creative Commons license, unless indicated otherwise in a credit line to the material. If material is not included in the article's Creative Commons license and your intended use is not permitted by statutory regulation or exceeds the permitted use, you will need to obtain permission directly from the copyright holder. To view a copy of this license, visit http://creativecommons.org/licenses/by/4.0/. 


\section{REFERENCES}

Barton, P.B. and Toulmin, P., Phase relations involving sphalerite in the $\mathrm{Fe}-\mathrm{Zn}-\mathrm{S}$ system, Econ. Geol., 1966, vol. 61, no. 5, pp. 815-849.

Dobrovol'skaya, M.G., Bortnikov, N.S., and Naumov, V.B., Fe mole fraction of sphalerite as indicator of sulfur regime during formation of ore deposits, Geol. Rud. Mestorozhd., 1991, no. 5, pp. 80-93.

Korzhinskii, D.S., Teoreticheskie osnovy analiza paragenezisov mineralov (Theoretical Principles of Analysis of Mineral Assemblages), Moscow: Nauka, 1973.

Lusk, J. and Calder, B.O.E., The composition of sphalerite and associated sulfides in reactions of the $\mathrm{Cu}-\mathrm{Fe}-\mathrm{Zn}-\mathrm{S}$, $\mathrm{Fe}-\mathrm{Zn}-\mathrm{S}$, and $\mathrm{Cu}-\mathrm{Fe}-\mathrm{S}$ systems at 1 bar and temperatures between 250 and $535^{\circ} \mathrm{C}$, Chem. Geol., 2004, vol. 203, no. 3, pp. 319-345.

Lyubimtseva, N.G., Bortnikov, N.S., Borisovsky, S.E., Prokofiev, V.Yu., and Vikent'eva, O.V., Fahlore and sphalerite from the Darasun gold deposit in the Eastern Transbaikal Region, Russia: I. Mineral assemblages and intergrowths, chemical composition, and its evolution, Geol. Ore Deposits, 2018a, vol. 60, no. 2, pp. 93-120.

Lyubimtseva, N.G., Bortnikov, N.S., Borisovsky, S.E., Prokofiev, V.Yu., and Vikent'eva, O.V., Fahlore and sphalerite from the Darasun gold deposit in the Eastern Transbaikal Region, Russia: II. Fe and Zn partitioning, fluid inclusions, and formation conditions, Geol. Ore Deposits, 2018b, vol. 60 , no. 3, pp. 220-240.

Lyubimtseva, N.G., Bortnikov, N.S., and Borisovsky, S.E., Coexisting bournonite-seligmannite and tennantite-tetrahedrite solid solutions of the Darasun Gold Deposit, East- ern Transbaikalia, Russia: estimation of the mineral formation temperature, Geol. Ore Deposits, 2019, vol. 61, no. 3, pp. 274-292.

Prokof'ev, V.Yu., Zorina, L.D., Baksheev, I.A., Plotinskaya, O.Yu., Kudryavtseva, O.E., and Ishkov, Yu.M., Minerals and formation conditions of ores of the Teremkin gold deposit (Eastern Transbaikal Region, Russia), Geol. Ore Deposits, 2004, vol. 46, no. 5, pp. 332-352.

Prokofiev, V.Y., Garofalo, P.S., Bortnikov, N.S., Kovalenker, V.A., Zorina, L.D., Grichuk, D.V., and Selektor, S.L., Fluid inclusion constraints on the genesis of gold in the Darasun district (Eastern Transbaikalia), Russia, Econ. Geol., 2010, vol. 105, no. 2, pp. 395-416.

Raabe, K.C. and Sack, R.O., Growth zoning in tetrahedrite-tennantite from the hock hocking mine, Alma, Colorado, Can. Mineral., 1984, vol. 22, pp. 577-584.

Sack, R.O. and Loucks, R.R., Thermodynamic properties of tetrahedrite-tennantite: constraints on the interdependence of the $\mathrm{Ag}-\mathrm{Cu}, \mathrm{Fe}-\mathrm{Zn}, \mathrm{Cu}-\mathrm{Fe}$, and $\mathrm{As}-\mathrm{Sb}$ exchange reactions, Am. Mineral., 1985, vol. 70, nos. 11-12, pp. $1270-1289$.

Scott, S.D. and Barnes, H.L., Sphalerite geothermometry and geobarometry, Econ. Geol., 1971, vol. 66, pp. 653-669. Sack, R.O., Fahlore thermochemistry: gaps inside the $(\mathrm{Cu}, \mathrm{Ag})_{10}(\mathrm{Fe}, \mathrm{Zn})_{2}(\mathrm{Sb}, \mathrm{As})_{4} \mathrm{~S}_{13}$ Cube, Petrology, 2017, vol. 25, no. 5, pp. 498-515.

Timofeevskii, D.A., Geologiya i mineralogiya Darasunskogo zolotorudnogo regiona (Geology and Mineralogy of the Darasun Gold District), Moscow: Nedra, 1972.

Translated by N. Astaf'ev 